\title{
PROPUESTA CURRICULAR PARA LA LICENCIATURA EN LA ENSEÑANZA DE LA FÍSICA, LA QUÍMICA Y LA BIOLOGÍA QUE OFRECE LA UNIVERSIDAD DE COSTA RICA
}

\author{
Leda María Roldán Santamaría
}

\section{Resumen}

El presente artículo recoge los resultados de una investigación realizada para determinar las necesidades de cambio en el plan de estudios de formación de licenciadas(os) en la Enseñanza de la Física, la Química y la Biología, que se gradúan en la Universidad de Costa Rica (UCR). Al final se presenta una propuesta curricular para lograr su actualización.

\section{Abstract}

This article is the report of the investigation about of the Academic program of Sciences' teacher, with speciality in physics, chemistry and biology in the University of Costa Rica. That program was presented in 1990 and in this moment it recoated changes. Finally it presents the new project for the academy program for Natural Science's teacher in physics, chemistry and biology.

\section{Palabras claves}

Enseñanza de las Ciencias: Física, Química y Biología. Investigación y formación de profesores de ciencias.

\section{$\mathrm{P}$} ara la integración del Currículo de una o un especialista en la Enseñanza de la Física, la Química y la Biología, se debe hacer especial énfasis en el avance de la ciencia y la tecnología, aspecto endógeno de gran relevancia en la formación del campo de la Enseñanza de las Ciencias y sus especialidades.

\footnotetext{
${ }^{1}$ Máster en Planificación Curricular. Universidad de Costa Rica. Licenciada en Física y Profesora de Física en esta universidad. Trabaja con el Instituto de Investigación en Educación. Iroldan @cariari.ucr.ac.cr
} 
También, se deben tener presentes las nuevas teorías educativas que conducen a que la o el estudiante de Licenciatura en la Enseñanza de la Física, la Química y la Biología, logre una mejor construcción de los conceptos y una mejor manera de facilitárselos a sus futuras(os) estudiantes.

Las(os) empleadoras(es) de estas(os) profesionales, representadas(os) por el Ministerio de Educación Pública, entidad directora del Sistema Educativo Costarricense, son las(os) que aportan las características que deben reunir estas(os) profesionales. Este ministerio proporciona directrices tanto a las instituciones de educación pública como a las de educación privada, que son las que las(os) contratan. Aquí se deben considerar los retos, la complejidad y los problemas del Sistema Educativo Costarricense.

\section{Lineamientos del Ministerio de Educación Pública}

El Ministerio de Educación Pública es el ente contralor de la educación costarricense. En su misión y visión de la actualización de la educación costarricense, presenta la siguiente posición con respecto a los programas de estudio:

"El umbral del tercer milenio ha traído consigo la revolución científicotecnológica, la globalización del planeta y la urgencia de hacer sostenible el desarrollo. Los países con índices positivos de desarrollo humano y altamente informatizado ya han comenzado a cimentar alianzas entre naciones, alianzas con la tecnología y con la naturaleza: todo en beneficio de su propio desarrollo. Es un cambio de paradigma en la forma de ver el mundo. Es la manera de buscar un nuevo humanismo, calidad de vida y competitividad.

Costa Rica no puede permanecer pasiva ante los acontecimientos mundiales. Debemos ser constructores de lo que pasa en este nuevo paradigma global. O nos ponemos a la delantera o tendremos severas dificultades para encontrar una posición digna en las alianzas que debemos procurar con las naciones, con las humanidades, las ciencias naturales y sociales, con la tecnología y con la naturaleza.

Para tomar esta delantera, todos los costarricenses - y muy especialmente los costarricenses del Siglo XXI que hoy son estudiantes de la Educación General Básica — debemos desarrollar una actitud activa ante el cambio y ante la forma en que pensamos sobre el mundo. Por un lado, es importante que, más que solamente adaptarnos, podamos anticiparnos a los cambios. Y por otro lado, es necesario que asumamos un pensamiento integrador. Indudablemente es a través de la educación que se construyen los conocimientos necesarios para el cambio de actitudes y el desarrollo del 
pensamiento. Por esto, y para responder en forma anticipada a estos grandes retos que enfrenta Costa Rica al finalizar el Siglo XX, es que el Ministerio de Educación Pública ha propuesto la Política Educativa hacia el Siglo XXI, que fue aprobada por el Consejo Superior de Educación el 8 de noviembre de 1994, la que propone una Ética del Desarrollo Sostenible y, con una visión humanista, reconoce la necesidad de la construcción y reconstrucción del pensamiento racional, científico y sistemático.

Seis elementos interrelacionados y coherentes componen la Política Educativa hacia el Siglo XXI. La acción mutua de estos seis elementos podrá contribuir a generar la educación que requiere el país para enfrentar las demandas del Siglo que se avecina: Programas de Estudio, Capacitación y Formación de Docentes, Evaluación, Libros de texto y otros recursos didácticos, Mediación docente, Intermediación gerencial" (MEP, 2002).

Ante esta visión de actualización que plantea el Ministerio de Educación Pública, se presentan nuevos programas de estudio acordes con el avance de la ciencia y la tecnología, lo que obliga a la actualización de las y los docentes en servicio, así como de los planes de formación de las(os) profesoras(es) que desean especializarse en las universidades.

\section{Enseñanza de las Ciencias}

Las nuevas propuestas que surgen de los organismos internacionales, que se preocupan por la educación de los países en vías de desarrollo, consideran que la Enseñanza de las Ciencias y sus especialidades debe estar cada día más acorde con las nuevas tecnologías y los avances científicos. Cada país debe tomar en cuenta sus características sociales, culturales y económicas, sus necesidades y sus problemas, para que sean la base de la definición de los objetivos, la selección de los contenidos y los problemas de trabajo, así como para las actividades de aprendizaje y evaluación, que se propongan en los programas de Enseñanza de las Ciencias (UNESCO, 1999).

Cuanto más enraizada esté la Enseñanza de las Ciencias en la problemática del país y más conexiones se establezcan con los problemas tecnológicos y las implicaciones sociales, más fácil resultará motivar a las(os) alumnas(os) y existirán más posibilidades de que sean capaces de transferir lo aprendido en el aula a su vida cotidiana.

No se conocen con certeza las necesidades del siglo XXI, pero si se considera lo que se logró en el siglo XX, se puede esperar que en este nuevo siglo se presenten más adelantos. La ciencia y la tecnología avanzan rápidamente, y 
en una nación en vías de desarrollo, los eventuales cambios se producirán a más largo plazo. Ante esta situación, la educación deberá proveer a la población aquellos elementos cognitivos necesarios para convivir con la tecnología, teniendo a la vista las posibles variantes de su manifestación cultural.

La tecnología ha cambiado, así como las comunicaciones; hoy se puede obtener un dato en el mismo tiempo que tarda en comunicarse telefónicamente, y más rápido aún. El ser humano cruza el océano, pero también lo hace a la Luna, instala tecnología que orbita la Tierra y es capaz de mandar naves a otros planetas.

Para buscar el significado de una palabra, hoy se usa una enciclopedia computarizada. Para obtener dinero de un banco, se puede introducir una tarjeta magnética en un cajero automático que funciona todos los días, durante las 24 horas. Se puede estar trotando y, al mismo tiempo, escuchar música con equipos miniaturizados de alta fidelidad, que transportan su propia fuente de energía. Se puede hablar por teléfono cuando se va cómodamente sentado en un bus o ver un partido de fútbol, en el momento en que se juega, aunque se esté a miles de kilómetros de distancia. Se puede pensar en vivir hasta los setenta, ochenta o más años aún. Una niña o un niño de 4 años, antes de aprender a saltar en una cuerda, puede aprender a usar un software computacional que varias(os) científicas(os) tardaron años en diseñarlo, con inversiones millonarias. Ante todos estos cambios, se hace necesario un viraje radical en la educación y en la Enseñanza de las Ciencias y de sus especialidades de manera particular.

La ciencia de hoy debe ser enseñada sin olvidar aspectos históricos importantes, pero hacerlo con el lenguaje apropiado, con una duración menos extensa (por idea o tema), con técnicas y métodos actuales, con tecnología apropiada, lo más vivencial que se pueda, con profesoras(es) que tengan un acercamiento con las y los estudiantes. Lo apropiado está determinado por las circunstancias históricas que envuelven a cada sector educacional. La educadora o el educador debe tener claridad sobre como enseñar y ser capaz de producir la interacción entre lo que ya se sabe y lo que se va a aprender. Tampoco, debe perder de vista el objetivo de este proceso, en el cual el protagonista es la o el estudiante, quien finalmente construirá sus conocimientos.

\section{Formación de especialistas en la Enseñanza de la Física, la Química y la Biología}

Para la formación integral de las y los especialistas en la Enseñanza de las Ciencias, se debe tener presente el perfil necesario para esa(e) futura(o) profesional de la educación. Ante una sociedad que ya no se conforma con el sistema 
educativo vigente y que clama por una mejor educación, se requieren docentes con un alto grado de profesionalismo y capacidad de actuación autónoma, sensibles para incorporar las demandas sociales a su programa educativo y aptas(os) para conseguir el equilibrio entre la comprensión y la atención a las diferencias individuales en el aula, así como para prevenir o superar manifestaciones de discriminación, violencia o enajenación. Deben ser profesionales capaces de tomar en cuenta las reformas de los programas, adaptarlas a las necesidades y limitaciones de su centro de trabajo.

Se debe considerar que estas(os) licenciadas(os) han de formarse para que puedan trabajar eficientemente en la Educación Media (Tercer Ciclo de Enseñanza General Básica y el Ciclo Diversificado), gracias al conocimiento pedagógico y de las ciencias básicas, al conocimiento de la didáctica general y específica y al desarrollo de actitudes que favorezcan el buen ejercicio docente, así como el mejoramiento de su grupo profesional y, en general, de la sociedad para la que laboran.

Ante lo anterior, los miembros de la Comisión Compartida de Enseñanza de las Ciencias, comisión que coordina dicha carrera en la Universidad de Costa Rica (1998 a 1999), consideran que las(os) licenciadas(os) en la Enseñanza de la Física, la Química y la Biología deben cumplir con los siguientes objetivos:

- Completar su formación en el campo de una especialidad científica.

- Adquirir nuevos conocimientos en las ciencias de la educación que las(os) capacite para desempeñar su función docente en forma más acertada y su papel de educadora o educador y facilitadora o facilitador del aprendizaje con mayores conocimientos.

- Adquirir conocimientos básicos para realizar investigaciones en el campo de la evaluación educativa y de la especialidad científica.

- Discernir, con mayores criterios, los métodos y técnicas a emplear en su propia situación de enseñanza y aprendizaje.

- Ejecutar investigaciones educativas que la(o) lleven a mejorar su labor docente.

- Emplear sus conocimientos en la especialidad en los campos del currículum, la didáctica y las teorías de aprendizaje en las situaciones reales de enseñanza y aprendizaje.

- Ampliar sus conocimientos teóricos y prácticos en el campo de su especialidad (Comisión Compartida de Enseñanza de las Ciencias, 1999). 


\section{Lineamientos de la Universidad de Costa Rica}

La Universidad de Costa Rica establece su misión y su visión en el Estatuto Orgánico. En este, el artículo 6 contempla, entre sus funciones, las siguientes:

“d) Formar un personal idóneo que se dedique a la enseñanza, las ciencias, las artes y las letras, para que participe eficazmente en el desarrollo del sistema de educación costarricense.

h) Formar profesionales en todos los campos del saber capaces de transformar, provechosamente para el país, las fuerzas productivas de la sociedad costarricense y de crear conciencia crítica en torno a los problemas de la dependencia y del subdesarrollo".

Estas funciones implican que la y el profesional que se gradúa en la Universidad de Costa Rica, como institución pública, responde a las demandas de la sociedad costarricense. La y el profesional en la Enseñanza de la Física, la Química y la Biología obtiene el grado de licenciada(o), grado académico que le brinda una categoría profesional superior, como educadora o educador especializada(o) en la enseñanza de una ciencia, y la(o) califica en un rango de desempeño laboral, según las exigencias del Sistema Educativo Costarricense.

En la modernización de su estructura, la Universidad de Costa Rica cuenta con vicerrectorías encargadas de velar por los diferentes campos del quehacer universitario. Le corresponde a la Vicerrectoría de Docencia, por su parte, velar por la actualización de los planes de estudio. Este aspecto lo contempla el Estatuto Orgánico en el capítulo 5, artículo 50, donde se indica lo siguiente:

“a) Sancionar y supervisar los diversos planes de estudio de la Universidad, con el propósito de coordinarlos en lo posible y adaptarlos a las necesidades de interés nacional.

b) Velar porque la labor docente en la Universidad se lleve a cabo en forma eficiente y actualizada, en unidad de propósitos con la investigación, utilizando los sistemas más adecuados de enseñanza y evaluación” (p. 14).

Como parte de sus funciones, la Vicerrectoría de Docencia vela por la administración y supervisión de los planes de estudio y sus correspondientes programas. Por otra parte, le ha correspondido emitir resoluciones relacionadas con el planteamiento, la evaluación, el control y las modificaciones de los planes, de acuerdo con los lineamientos de la reglamentación universitaria establecida en su Estatuto Orgánico y las directrices que dicta el Consejo Nacional de Rectores (CONARE). 
En el documento sobre Políticas y Normas Curriculares para la Actualización de Planes de Estudio (1995), emitido por la Vicerrectoría de Docencia, y en la posición curricular de la Universidad de Costa Rica, es posible establecer tres niveles esenciales. El primero es el orientador, que comprende los principios, propósitos y políticas institucionales; el segundo corresponde a las regulaciones establecidas en la normativa, donde se incluyen los reglamentos y resoluciones, y el tercero comprende los planes y programas de estudio en los que se definen los aprendizajes que las(os) futuras(os) profesionales deben lograr para conseguir un grado académico.

Las facultades que forman parte y son responsables de la formación de licenciadas(os) en la Enseñanza de la Física, la Química y la Biología, son las facultades de Ciencias y Educación. De acuerdo con el reglamento de la Facultad de Ciencias, tiene como parte de sus fines el siguiente:

“c) Preparar profesionales para la Enseñanza de las Ciencias Básicas en la Educación Media, en forma coordinada con la Facultad de Educación".

La Facultad de Ciencias está integrada por cinco unidades académicas que, en relación con el plan de estudios, contribuyen en la formación de las(os) profesoras(es) de ciencias, aportando los conocimientos científicos:

- La Escuela de Física brinda el conocimiento en el campo de las ciencias físicas, de la astronomía y la meteorología.

- La Escuela de Química da formación en el campo de la química general, la orgánica, la inorgánica y otros campos propios de esa ciencia.

- La Escuela de Biología ofrece el conocimiento en los campos de la Biología general, la Botánica, la Zoología, la Genética y otras ciencias afines.

- La Escuela de Geología forma en el campo de la geología general y los desastres naturales.

- La Escuela de Matemática brinda las herramientas necesarias en el campo del cálculo infinitesimal e integral, conocimientos necesarios en la solución de problemas del área científica.

La Facultad de Educación tiene como misión formar profesionales en diferentes especialidades de la Educación, con el propósito de atender las demandas de nuestro sistema educativo. Es la responsable de la formación pedagógica y de otros aspectos afines a la labor profesional de las(os) profesoras(es) de enseñanza media. 
La Facultad de Educación cuenta con tres unidades académicas:

- Administración Educativa, que brinda el curso de formación en el uso de los recursos audiovisuales y la administración educativa.

- Formación Docente, da formación en los campos pedagógico, curricular, didáctico y de investigación educativa.

- Orientación y Educación Especial, brinda los cursos para la formación psicopedagógica, de evaluación y necesidades educativas especiales.

Todas estas unidades académicas, como parte de la Universidad de Costa Rica, son las responsables de la formación de las(os) licenciadas(os) en la Enseñanza de la Física, la Química y la Biología.

\section{Perfil profesional}

El actual plan de estudios de Licenciatura en la Enseñanza de la Física, la Química y la Biología presenta un perfil profesional acorde con la formación de profesionales especializadas(os) en la Enseñanza de las Ciencias y sus especialidades.

El diagnóstico evidencia la necesidad de formar una(un) profesional actualizada(o) y con los conocimientos psicopedagógicos apropiados para la población que debe atender, por lo que esta(e) debe fortalecerse.

En esta propuesta, se define un perfil profesional, según las nuevas tendencias educativas expresadas en el informe Delors (1996), al considerar los cuatro pilares de la educación (aprender a conocer, aprender a hacer, aprender a vivir y aprender a ser). Las mismas tendencias se consideraron para la propuesta de la o el Bachiller en la Enseñanza de las Ciencias Naturales y se retoman aquí, ya que se debe tener congruencia en la formación de profesionales especializadas(os) en la Enseñanza de las Ciencias.

La Escuela de Formación Docente, en la evaluación del núcleo pedagógico, consideró esos cuatro pilares para definir el perfil de la(el) egresada(o) de este núcleo (Carvajal y otros, 1999, anexo 2). Esas divisiones establecidas para el perfil de la(del) egresada(o) se toman en cuenta en esta propuesta. Con este perfil se espera que la(el) licenciada(o) en la Enseñanza de la Física, la Química y la Biología reúna los cuatro pilares:

\section{Aprender a conocer}

- Reconoce las características sociales, culturales, políticas y económicas de la realidad costarricense. 
- Establece relaciones entre las teorías pedagógicas y el contexto educativo del aula.

- Considera el análisis de los fundamentos filosóficos, antropológicos, políticos y culturales de las teorías pedagógicas que orientan su labor docente.

- Se actualiza con respecto a las nuevas teorías pedagógicas, metodológicas y didácticas.

- Consolida su formación psicopedagógica para el nivel de estudiantes que deberá atender en su desarrollo profesional.

- Fundamenta su labor docente en principios teóricos de enseñanza y aprendizaje.

- Desarrolla destrezas de autoaprendizaje.

- Fortalece su conocimiento en los campos de la Física, la Química, la Biología y las áreas afines.

\section{Aprender a hacer}

- Integra los conocimientos científicos, destrezas y habilidades propias de su disciplina en la planificación, ejecución y evaluación de su labor docente.

- Hace uso de la autoevaluación como un recurso para retroalimentar su labor.

- Establece relaciones entre la teoría y la práctica pedagógica para innovar en el aula.

- Desarrolla actividades docentes, investigativas y de acción social.

- Utiliza la investigación y la acción social para fortalecer su labor docente.

- Utiliza estrategias metodológicas de acuerdo con criterios curriculares.

- Ajusta los programas y las actividades de enseñanza y aprendizaje a las necesidades y expectativas de las(os) educandas(os).

- Estimula en sus estudiantes la investigación, tanto en el área social como en el área científica.

- Hace uso de los recursos tecnológicos a su alcance para mejorar el proceso de enseñanza y aprendizaje.

- Atiende las necesidades curriculares y extracurriculares de sus estudiantes con sus aspectos educativos especiales.

\section{Aprender a vivir}

- $\quad$ Promueve su participación y la de las(os) educandas(os) en experiencias interdisciplinarias. 
- Participa con sus colegas y estudiantes en actividades de bien común.

- Estimula en sus educandas(os) una actitud de respeto hacia el ambiente.

- Hace evidente su interés y responsabilidad por la preservación de los recursos naturales.

- $\quad$ Promueve relaciones de respeto entre colegas, estudiantes, padres de familia y miembros de la comunidad.

\section{Aprender a ser}

- Se interesa por ejercer de manera autónoma y responsable la misión docente en el marco institucional y comunitario.

- Se manifiesta como líder para conducir el cambio y la innovación educativa.

- Participa en las actividades que conduzcan a un mayor desarrollo profesional.

- Estimula en la(el) educanda(o) el desarrollo del pensamiento crítico ante los cambios científicos y tecnológicos.

- Hace evidente su formación humanística y cultural, que responde a los lineamientos establecidos por la Universidad de Costa Rica.

- $\quad$ Es creativa(o) en el diseño, ejecución y evaluación de su tarea docente.

- Desarrolla una actitud crítica y científica que promueva la resolución de problemas.

La(el) licenciada(o) en la Enseñanza de la Física, la Química y la Biología debe estimular, mediante la enseñanza y el aprendizaje de las ciencias, la construcción de conceptos en las Ciencias Naturales y sus especialidades, que permitan derivar conclusiones útiles para enfrentar la vida con actitudes creativas y racionales, que favorezcan el logro de destrezas, habilidades, valores, actitudes y principios conceptuales que contribuyan al desarrollo integral del individuo, así como el manejo de nuevas tecnologías y el uso crítico de la información.

La Enseñanza de las Ciencias y sus especialidades también promueven la práctica de procedimientos, valores y actitudes propios del quehacer científico, como la autodisciplina, la responsabilidad, el respeto y la tolerancia, el aprecio por la comprobación de los hechos, la resolución de problemas y la objetividad en la búsqueda de explicaciones razonables; aspectos necesarios para comprender la realidad personal y ubicarse en el contexto ambiental.

La educación científica debe propiciar una relación más justa entre los seres humanos y la naturaleza, promover la utilización adecuada de los elementos 
naturales, requisitos indispensables para la supervivencia de la humanidad, su calidad de vida y el desarrollo sostenible de nuestra región.

\section{Objetivos del plan de Licenciatura en Enseñanza de la Física, la Química y la Biología}

El plan de Licenciatura en la Enseñanza de la Física, la Química y la Biología proporcionará a la y el estudiante los medios necesarios para:

1. Completar su formación en el campo de una especialidad científica.

2. Realizar investigaciones en la Enseñanza de las Ciencias.

3. Planear y efectuar investigaciones educativas para mejorar su labor docente.

4. Ejecutar labores de planeamiento curricular.

5. Basar sus decisiones curriculares y didácticas en el análisis serio de los factores que influyen en la docencia.

6. Adquirir nuevos conocimientos en las ciencias de la educación, que las(os) capacite mejor para desempeñar su función docente.

7. Adquirir conocimientos básicos para realizar investigaciones en el campo de la evaluación educativa y de la especialidad científica.

8. Discernir mejor los métodos y técnicas a emplear en su propia situación de enseñanza y aprendizaje.

9. Ejecutar investigaciones educativas que la(o) lleven a mejorar su labor docente.

10. Emplear sus conocimientos en la especialidad en los campos del currículum, la didáctica y las teorías de aprendizaje en las situaciones reales de enseñanza y aprendizaje.

11. Ofrecer a la sociedad costarricense una y un profesional en Ciencias que se mantenga actualizada(o) en cuanto a los avances y tendencias del pensamiento científico-tecnológico, educativo, cultural y social.

\section{Malla curricular}

Para ingresar a la Licenciatura en la Enseñanza de la Física, la Química y la Biología, se deben cumplir los siguientes requisitos, que reúnen cualquiera de las siguientes posibilidades:

a) Bachillerato en la Enseñanza de las Ciencias con énfasis en Física, Química o Biología. 
b) Bachillerato en Ciencias de la Educación con énfasis en Enseñanza Media y título de Profesor de Enseñanza Media en Biología, Física o Química.

c) Bachillerato en la Enseñanza de las Ciencias y Bachillerato en Física, Química o Biología.

d) Bachillerato en Física, Química o Biología y Profesor en Enseñanza Media en Física, Química o Biología.

e) Bachillerato en Enseñanza de las Ciencias Naturales.

En la siguiente malla curricular, se puede apreciar que los cursos se han ordenado de acuerdo con el área del conocimiento. Su distribución obedece a la secuencia de los cursos requisitos, respetando que en cada ciclo se logre acomodar un máximo de 18 créditos. En el área de educación, se propone un curso electivo, el cual se puede escoger entre los siguientes:

Sigla

Nombre del curso

Créditos

EA-0379

Tecnología educativa 3

EA-0399

Epistemología educativa

FD-0467

Legislación educativa

AS-1102

Etnología de la educación

OE-

Necesidades educativas especiales*

\footnotetext{
* Curso que debe crearse por parte de la Escuela de Orientación y Educación Especial.
}

Los cursos de las especialidades se proponen de acuerdo con las ofertas que cada unidad académica está ofreciendo, con la idea de no generar gastos administrativos con la creación de nuevos cursos. Se debe tener presente que esta licenciatura es para formar mejores docentes y más especializados en la Enseñanza de las Ciencias, de la Física, la Química y la Biología; no pretende formar licenciadas(os) en Física, Biología o Química.

Los cursos propuestos en la malla curricular pueden ser sustituidos según sugerencia de las unidades académicas y de acuerdo con la demanda que los cursos tengan. El curso de Amenazas Naturales y Desastres se propone para las tres licenciaturas, ya que la formación de las(os) profesoras(es) de ciencias en este campo presenta déficit en esta área, por lo menos las(os) que son formadas(os) en la Universidad de Costa Rica. 
Una vez aprobados los cursos del plan de estudios, la o el estudiante debe ganar al menos dos cursos de investigación dirigida que le permitan la preparación de su investigación final, según los proyectos finales de graduación, donde puede seguir cualquiera de las modalidades que la Universidad de Costa Rica ofrece para estos casos.

En la siguiente malla curricular se aprecian los cursos por áreas y sus respectivos requisitos y correquisitos.

\begin{tabular}{|c|c|c|c|c|c|}
\hline \multirow{2}{*}{$\begin{array}{c}\text { Áreas del } \\
\text { conocimiento }\end{array}$} & \multicolumn{2}{|c|}{ I CICLO } & \multicolumn{3}{|c|}{ II CICLO } \\
\hline & $\begin{array}{c}\text { Métodos de } \\
\text { investigación } \\
\text { educativa } \\
\text { FD-5091 }\end{array}$ & $\begin{array}{c}\text { Planeamiento } \\
\text { y desarrollo } \\
\text { curricular } \\
\text { FD-8091 }\end{array}$ & $\begin{array}{c}\text { Análisis } \\
\text { cuantitativo } \\
\text { paramétrico en } \\
\text { educación } \\
\text { FD-5111 }\end{array}$ & $\begin{array}{c}\text { Seminario en } \\
\text { la Enseñanza } \\
\text { de la Física, la } \\
\text { Química o la } \\
\text { Biología } \\
\text { FD-5100 }\end{array}$ & Electiva \\
\hline Créditos & (3) & (3) & (3) & (3) & (3) \\
\hline Requisito & & & FD-5091 & & \\
\hline $\begin{array}{c}\text { Física } \\
\text { (13 créditos) }\end{array}$ & $\begin{array}{l}\text { Termodinámica } \\
\text { FS-0408 }\end{array}$ & $\begin{array}{l}\text { Cálculo } 3 \\
\text { MA-1003 }\end{array}$ & $\begin{array}{c}\text { Física General } \\
\text { III } \\
\text { FS-0410 }\end{array}$ & $\begin{array}{c}\text { Laboratorio de } \\
\text { Física General } \\
\text { III } \\
\text { FS-0411 }\end{array}$ & $\begin{array}{c}\text { Amenazas } \\
\text { Naturales y } \\
\text { Desastres } \\
\text { G-0045 }\end{array}$ \\
\hline Créditos & (3) & (3) & (3) & (1) & (3) \\
\hline Requisitos & $\begin{array}{l}\text { FS-0310, } \\
\text { FS-0311 } \\
\text { MA-2210 }\end{array}$ & MA-2210 & $\begin{array}{l}\text { FS-0310, } \\
\text { FS-0311 } \\
\text { MA-1003 }\end{array}$ & $\begin{array}{l}\text { FS-0310, } \\
\text { FS-0311 }\end{array}$ & \\
\hline $\begin{array}{c}\text { Química } \\
\text { (15 créditos) }\end{array}$ & $\begin{array}{c}\text { Química } \\
\text { analítica } \\
\text { cuantitativa I } \\
\text { QU-0200 }\end{array}$ & $\begin{array}{c}\text { Laboratorio de } \\
\text { Química } \\
\text { Analítica } \\
\text { QU-0201 }\end{array}$ & $\begin{array}{l}\text { Tópicos de } \\
\text { Química I } \\
\text { QU-0202 }\end{array}$ & $\begin{array}{c}\text { Físico-química } \\
\text { para ingeniería } \\
\text { química } \\
\text { QU-0260 }\end{array}$ & $\begin{array}{c}\text { Amenazas } \\
\text { Naturales y } \\
\text { Desastres } \\
\text { G-0045 }\end{array}$ \\
\hline Créditos & (3) & (2) & (3) & (4) & (3) \\
\hline Requisitos & $\begin{array}{l}\text { QU-0102 } \\
\text { QU-0103 }\end{array}$ & $\begin{array}{l}\text { QU-0102 } \\
\text { QU-0103 }\end{array}$ & $\begin{array}{l}\text { QU-0102 } \\
\text { QU-0103 }\end{array}$ & $\begin{array}{l}\text { QU-0102 } \\
\text { FS-0310 } \\
\text { FS-0311 }\end{array}$ & \\
\hline $\begin{array}{c}\text { Biología } \\
\text { (18 créditos) }\end{array}$ & $\begin{array}{l}\text { Historia } \\
\text { Natural de } \\
\text { Costa Rica } \\
\text { B-0300 }\end{array}$ & $\begin{array}{l}\text { Problemas } \\
\text { especiales en } \\
\text { Biología } \\
\text { B-0350 }\end{array}$ & $\begin{array}{l}\text { Introducción a } \\
\text { la Flora de } \\
\text { Costa Rica } \\
\text { B-0333 }\end{array}$ & $\begin{array}{l}\text { Curso a } \\
\text { proponer por } \\
\text { la Es. de } \\
\text { Biología }\end{array}$ & $\begin{array}{c}\text { Amenazas } \\
\text { Naturales y } \\
\text { Desastres } \\
\text { G-0045 }\end{array}$ \\
\hline Créditos & (4) & (4) & (4) & (3) & (3) \\
\hline Requisitos & B-0218 & & B-0239 & & \\
\hline
\end{tabular}


Para optar por el grado de Licenciada(o) en la Enseñanza de la Física, la Química y la Biología, la o el estudiante debe cumplir con los cursos del núcleo pedagógico y el núcleo de la especialidad. Por último, debe cumplir con el requisito de la investigación dirigida para concluir con este plan de estudios.

Esta propuesta curricular se presentó a la Comisión Compartida de Enseñanza de las Ciencias de la Universidad de Costa Rica y se aprobó para que continúe con el trámite correspondiente ante las autoridades universitarias. De aprobarse por parte de las autoridades respectivas, el plan entrará en vigencia en el primer ciclo del año 2006.

El estudio investigativo que dio origen a esta propuesta fue dirigido por la autora y apoyado por el Instituto de Investigación en Educación (INIE) de la Universidad de Costa Rica y por la Vicerrectoría de Investigación de la misma Universidad. El avance del estudio se dio a conocer en el Congreso Internacional de Educación que se efectuó en la Universidad de Costa Rica, en febrero del 2005, que coordinó el INIE. 


\section{Referencias}

Carvajal, C. y otros. (1999). Orientaciones técnico-curriculares para el proceso de revisión del núcleo pedagógico. Escuela de Formación Docente. UCR. San José, Costa Rica.

Comisión Compartida de Enseñanza de las Ciencias. (1999). Informe de revisión. UCR. San José, Costa Rica.

Delors, J. (1996). La educación encierra un tesoro. Espasa Calpe. Madrid.

MEP. (2002). Programa de Mejoramiento de la Calidad de la Educación General Básica. www.mep.go.cr/promece.asp

Roldán, L. (2002). Informe final: Propuesta curricular para actualizar el plan de estudios ofrecido por la Universidad de Costa Rica para la carrera de bachillerato en la enseñanza de las ciencias naturales. Trabajo presentado para optar por el grado de Máster en Planificación Curricular. UCR. San José, Costa Rica.

UNESCO. (1999). Reunión Regional de Consulta de América Latina y el Caribe. Conferencia Mundial sobre la Ciencia. República Dominicana. Anuies.mx/anuies/ revsup/res 110

Universidad de Costa Rica. (2000). Estatuto Orgánico. Oficina de Publicaciones. San José, Costa Rica.

Vicerrectoría de Docencia. (1995). Políticas y normas curriculares para la actualización de planes de estudio. UCR. San José, Costa Rica. 